\title{
Isospin Mixing Within Relativistic Mean-Field Models Including the Delta Meson
}

\author{
Clebson Abati Graeff ${ }^{* \dagger}$ \\ Grupo de Física Nuclear - Departamento de Física - Universidade Federal de Santa Catarina \\ E-mail: cgraeffefsc.ufsc.br
}

\section{J. R. Marinelli}

Grupo de Física Nuclear - Departamento de Física - Universidade Federal de Santa Catarina

E-mail: ricardo@fsc.ufsc.br

\begin{abstract}
We investigate isospin mixing effects in the asymmetry as obtained in parity-violating electron scattering for selected spherical nuclei ranging from ${ }^{4} \mathrm{He}$ to ${ }^{208} \mathrm{~Pb}$. The scattering analysis is developed within plane and distorted wave Born approximations (PWBA \& DWBA) accounting for nucleon form factors. The nuclear targets are described by a relativistic model Lagrangian with density dependent couplings, including the $\sigma, \omega, \rho$ and $\delta$ mesons as well as the electromagnetic interaction. The $\delta$ meson effects are specially interesting as they should add a contribution for isospin mixing together with the electromagnetic and $\rho$ meson fields. The system is solved in a Hartree approximation using a self-consistent calculation. We use two different parametrizations, one including the $\delta$ meson and another one in which it is not included. In this way, the effects of the inclusion of that meson can be inferred.
\end{abstract}

XXXIV edition of the Brazilian Workshop on Nuclear Physics

5-10 June 2011

Foz de Iguaçu, Paraná state, Brasil

\footnotetext{
*Speaker.

${ }^{\dagger}$ Supported by $\mathrm{CNPq}$
} 


\section{Introduction}

Parity Violating Electron Scattering (PVE) is a unique technique for separating the effects of the weak and the electromagnetic interactions between high energy projectiles and hadronic targets. In fact, the combination of polarized and non polarized electron beams can make that separation in a model independent way. This is possible since the electromagnetic interaction is independent of the polarization state of the incident particle, which is not the case for the weak interaction. In this way, we can define the asymmetry $\mathscr{A}$ as the ratio between the difference and the sum of the scattering cross-sections for beams with positive and negative polarizations.

For nuclei with an even number of protons $(Z)$ and neutrons $(N)$ in a Plane Wave Born Approximation (PWBA), the asymmetry has the form [1]

$$
\mathscr{A} \propto \sin ^{2} \theta_{W}\left[1+R_{V}^{T=0}+\Gamma(q)\right]+\left[1+R_{V}^{0}\right] \frac{G_{E}^{s}}{G_{E}^{0}} .
$$

The terms $R_{V}^{T=0}$ and $R_{V}^{0}$ are radiative corrections for the Standard Model and $\theta_{W}$ is the Weinberg angle. The term $\Gamma(q)$ accounts for the isospin mixing, i.e., contributions related to differences between proton and neutron distributions in the nucleus. Finally, $G_{E}^{s} / G_{E}^{0}$ represents the ratio of the nucleon strangeness form factor to the nucleon isoscalar form factor.

While the nuclei properties for low momentum transfer are satisfactorily explained using $u$ and $d$ quarks, for higher momentum transfer, excitations like the creation of quark-antiquark pairs may happen. Those particle-antiparticle pairs may give rise to significant effects in the interactions between the probe and the nucleons. The lower mass of the $s$ quark, when compared with $c, b$ and $t$ quarks, should imply in a dominant presence of $s \bar{s}$ pairs, which originates the strangeness form factor $G^{s}$. In an attempt to determine this form factor, measurements have been made using ${ }^{1} \mathrm{H}$ and ${ }^{4}$ He targets [2,3].

When interactions that differentiate protons from neutrons are not taken into account for $N=Z$ nuclei and the radiative corrections as well as the strangeness content are disregarded, the asymmetry is directly related to $\sin ^{2} \theta_{W}$ and can be used as a test of the Standard Model. Experiments using ${ }^{12} \mathrm{C}$ with this goal are already completed [4] and new ones using ${ }^{1} \mathrm{H}$ targets are in progress [5]. However, the presence of the electromagnetic interaction makes the isospin symmetry only an approximation, and a good understanding of the isospin mixing $\Gamma(q)$ is very important in order to correctly analyze the asymmetry measurements.

We shall be concerned here mainly with the effects of isospin mixing, based on a relativistic model for the nucleus, while ignoring any effects due to strangeness content or corrections originating from higher approximations in the Standard Model. Furthermore, only spherical nuclei in their ground states are considered. An important consequence of that isospin mixing is the relation between the asymmetry and the so called neutron skin, whose measurement is now under way for ${ }^{208} \mathrm{~Pb}$. We also present results using our approach for this and other closed-shell nuclei.

\section{Nuclear Model}

The model chosen for the nuclear interactions is based on Walecka's Model [6]. The interactions include the exchange of $\sigma$ (scalar), $\omega$ (vector), $\rho$ (isovector) and $\delta$ (isoscalar) mesons and the 
electromagnetic interaction. We start from a realistic Lagrangian which contains density dependent couplings (DD)

$$
\begin{aligned}
\mathscr{L}_{D D}= & \bar{\Psi}\left\{i \gamma^{\mu} \partial_{\mu}-m\right\} \Psi \\
& +\frac{1}{2} \partial^{\mu} \sigma \partial_{\mu} \sigma-\frac{1}{2} m_{\sigma}^{2} \sigma^{2}+\bar{\Psi} \Gamma_{\sigma} \sigma \Psi \\
& +\frac{1}{2} \partial_{\mu} \vec{\delta} \cdot \partial^{\mu} \vec{\delta}-\frac{1}{2} m_{\delta}^{2} \vec{\delta}^{2}+\bar{\Psi} \Gamma_{\delta} \vec{\tau} \cdot \vec{\delta} \Psi \\
& -\frac{1}{4} \Omega_{\mu v} \Omega^{\mu v}+\frac{1}{2} m_{\omega}^{2} \omega_{\mu} \omega^{\mu}-\bar{\Psi} \Gamma_{\omega} \gamma^{\mu} \omega_{\mu} \Psi \\
& -\frac{1}{4} \vec{R}_{\mu \nu} \vec{R}^{\mu v}+\frac{1}{2} m_{\rho}^{2} \vec{b}_{\mu} \cdot \vec{b}^{\mu}-\bar{\Psi} \Gamma_{\rho} \gamma^{\mu} \vec{\tau} \cdot \vec{b}_{\mu} \Psi \\
& -\frac{1}{4} F_{\mu v} F^{\mu v}-e \bar{\Psi} \frac{1-\tau_{3}}{2} A^{\mu} \Psi,
\end{aligned}
$$

with $\Omega_{\mu \nu} \equiv \partial_{\mu} \omega_{v}-\partial_{v} \omega_{\mu}, \vec{R}_{\mu \nu} \equiv \partial_{\mu} \vec{b}_{v}-\partial_{v} \vec{b}_{\mu}, F_{\mu \nu} \equiv \partial_{\mu} A_{v}-\partial_{v} A_{\mu}, \rho=\sqrt{J^{\mu} J_{\mu}}$ and $J^{\mu}=\bar{\Psi} \gamma^{\mu} \Psi$.

The Dirac and Klein-Gordon equations of motion obtained from the Euler-Lagrange equation for nucleons and mesons, respectively, are solved in a mean-field approach. Furthermore, we will consider only the static and spherically symmetric case. The resulting equations are then solved self-consistently using an oscillator basis expansion.

\section{Symmetry Energy \& Nuclear Properties}

In our analysis, we use two parameters sets: one in which the $\delta$ meson is not included - given by the TW parametrization [7] - and another in which it is included. The addition of the $\delta$ meson using the so called DDH $\delta$ [8] parametrization, which we have used in the past [9], does not reproduce the symmetry energy inside of the widely accepted range of 28 to $36 \mathrm{MeV}$. As is well known, a strong correlation has been established between the symmetry energy at saturation density and the neutron skin (see for instance reference [10]), making the correct reproduction of that property a very important feature in our analysis. For this reason, we seek a new set of parameters of the $\mathrm{DDH} \delta$ type, modified to better reproduce the binding energy and root mean square charge radius of spherical nuclei between ${ }^{16} \mathrm{O}$ and ${ }^{208} \mathrm{~Pb}$. As shown below, that new parameter set (DDH $\delta_{1}$ ) gives us a more reliable symmetry energy value and so a more confident result for the neutron skin and asymmetries.

In Table 1 we display our symmetric nuclear matter results at the saturation point for TW, $\mathrm{DDH} \delta$ and $\mathrm{DDH} \delta_{1}$. We emphasize that this last set was generated by fitting the binding energy and charge radius of spherical nuclei across the entire periodic table. In Table 2, binding energy, proton and neutron radius are shown for ${ }^{12} \mathrm{C}$ and ${ }^{208} \mathrm{~Pb}$. The first one is also included due to practical interest, once previous calculations and measurements of the asymmetry has been done for this nucleus. Finally, in Table 3 we show the neutron skin values obtained for all nuclei considered in this work.

In the mean-field calculation used to obtain the nuclear densities, the nucleons are taken as point-like. In order to account for their finiteness a correction should be made using nucleon form factors. Moreover, mean-field techniques do not take in to account the center-of-mass (CM) motion 


\begin{tabular}{cccc}
\hline & TW [7] & DDH $\delta[8]$ & DDH $\delta_{1}$ \\
\hline Sym. Energy $(\mathrm{MeV})$ & 32.77 & 25.73 & 32.70 \\
Compressibility $(\mathrm{MeV})$ & 240.2 & 240.2 & 244.4 \\
Saturation Density $\left(\mathrm{fm}^{-3}\right)$ & 0.153 & 0.153 & 0.147 \\
\hline
\end{tabular}

Table 1: Symmetry energy and compressibility for different parametrizations.

\begin{tabular}{|c|c|c|c|c|c|c|c|c|}
\hline & \multicolumn{4}{|c|}{${ }^{12} \mathrm{C}$} & \multicolumn{4}{|c|}{${ }^{208} \mathrm{~Pb}$} \\
\hline & TW & $\mathrm{DDH} \delta$ & $\mathrm{DDH} \delta_{1}$ & Exp. & TW & $\mathrm{DDH} \delta$ & $\mathrm{DDH} \delta_{1}$ & Exp. \\
\hline$E / A$ & -7.025 & -7.025 & -7.089 & $7.680[11]$ & -7.795 & -8.025 & -7.912 & $-7.867[11]$ \\
\hline$r_{p}$ & 2.396 & 2.400 & 2.442 & - & 5.466 & 5.490 & 5.541 & $5.50[12]$ \\
\hline$r_{n}$ & 2.372 & 2.368 & 2.414 & - & 5.660 & 5.613 & 5.724 & - \\
\hline
\end{tabular}

Table 2: Results for nuclear properties obtained by different parametrizations. The results for radii include the center of mass and nucleon form factors corrections.

\begin{tabular}{cccccccc}
\hline & ${ }^{12} \mathrm{C}$ & ${ }^{16} \mathrm{O}$ & ${ }^{40} \mathrm{Ca}$ & ${ }^{48} \mathrm{Ca}$ & ${ }^{66} \mathrm{Ni}$ & ${ }^{90} \mathrm{Zr}$ & ${ }^{208} \mathrm{~Pb}$ \\
\hline $\mathrm{TW}$ & -0.0249 & -0.0285 & -0.0504 & 0.1837 & 0.1497 & 0.0832 & 0.1940 \\
$\mathrm{DDH} \delta_{1}$ & -0.0282 & -0.0306 & -0.0531 & 0.1678 & 0.1500 & 0.0703 & 0.1829 \\
\hline
\end{tabular}

Table 3: Results for neutron skin calculated from corrected proton and neutron radii.

properly, so a harmonic oscillator correction is used as an approximation. Those corrections are written in a simpler way in momentum transfer $(q)$ space and are given by:

$$
\rho_{i}^{\prime}(q)=\rho_{i}(q) F_{C M}(q) F_{i}(q)
$$

where $\rho_{i}(q)$ is the point-like nucleon density $(i=p, n), F_{C M}(q)=\exp \left(q^{2} b^{2} / 4 A\right)$ and the nucleon form factors $F_{i}(q)$ are given by the the Galster parametrization [1]. For the proton density, we employ the Dirac $F_{1}$ nucleon electromagnetic form factor, while for the neutron density the neutral current $\tilde{F}_{1}$ form factor is used.

\section{Asymmetry}

The asymmetry can be defined in terms of the electron-nucleus cross-sections as

$$
\mathscr{A}=\frac{d \sigma_{+} / d \Omega-d \sigma_{-} / d \Omega}{d \sigma_{+} / d \Omega+d \sigma_{-} / d \Omega}
$$

where $d \sigma_{+} / d \Omega$ and $d \sigma_{-} / d \Omega$ stands for positive and negative helicity cross-sections. Evaluating this expression in a plane wave approximation (PWBA), the asymmetry takes a remarkably simple form:

$$
\mathscr{A}=\frac{\sqrt{2} G b}{4 \pi e} \frac{\mathscr{J}_{0}^{v *}(q)}{J_{0}(q)} q^{2}
$$




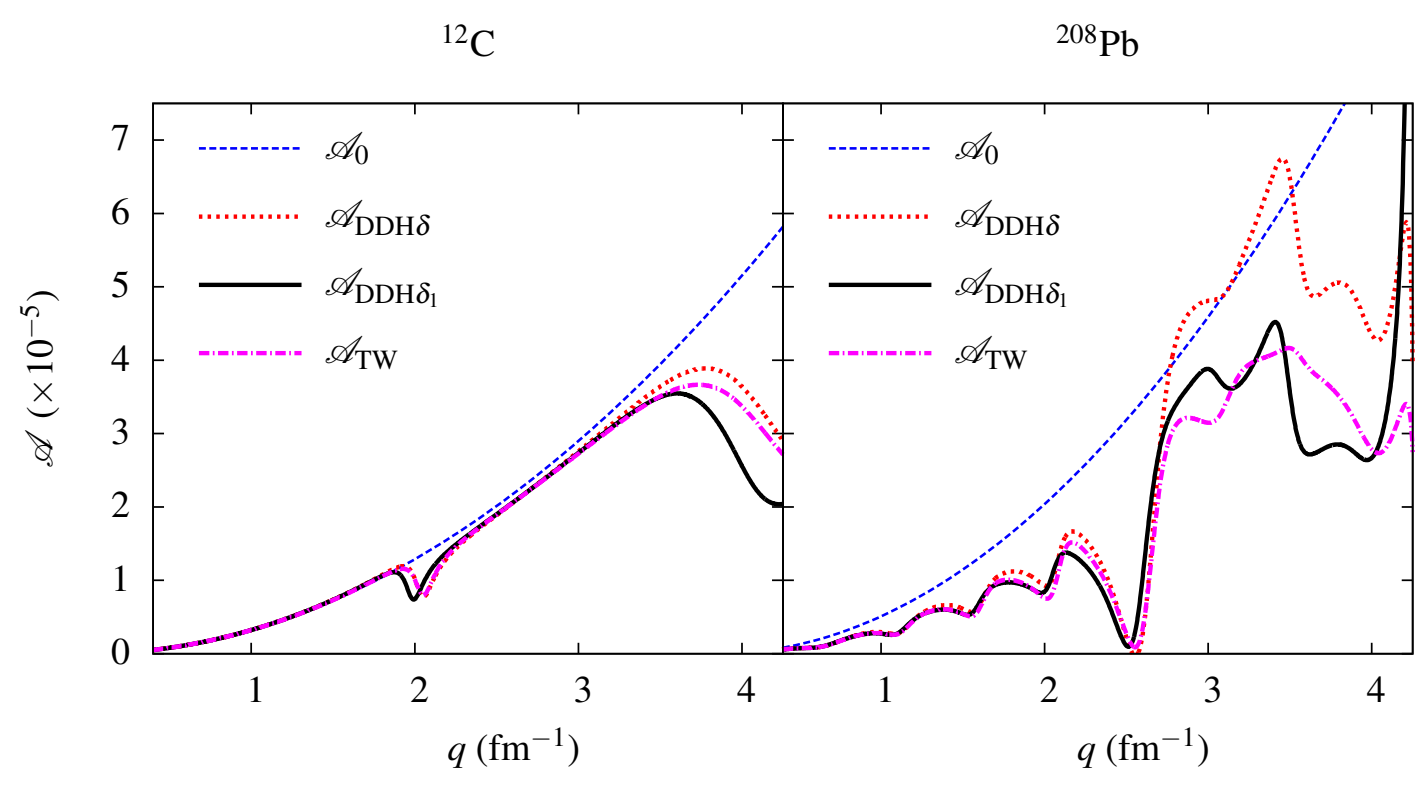

Figure 1: Results for ${ }^{12} \mathrm{C}$ and ${ }^{208} \mathrm{~Pb}$ asymmetry in DWBA for Density Dependent Hartree (DDH) parametrizations. Here $\mathscr{A}_{0}$ is given by equation (4.2), in the limit $\rho^{p}(q) / \rho^{n}(q)=Z / N$.

where the currents $J_{0}(q)$ and $\mathscr{J}_{0}^{v *}(q)$ stands for the electromagnetic and neutral currents, respectively, in $q$ space and can be written in terms of the densities $\rho_{i}^{\prime}(q)$. $G$ represents the Fermi constant for the weak force and $b$ is a characteristic electron weak interaction constant. These currents are then corrected by the nucleon form factors and approximate $\mathrm{CM}$ form factor. The calculation in the distorted wave approximation (DWBA), on the other hand, is performed by expanding the scattered electron wave functions in partial waves and solving numerically to obtain the cross-sections and the asymmetries. The inputs for those more involved calculations are the charge and weak neutral distributions, which also are corrected by CM and nucleon form factors. The results for the DWBA case are shown in Figure 4.

\section{Conclusions}

Relativistic models for the nucleus are know to give large values for the neutron skin, as compared to non-relativistic model calculations [13] and to the experimental values, even when the present large error bars are taken into account. In this sense, we seek to introduce the $\delta$ meson as it is expected to reduce the difference between proton and neutron radii distributions, consequently reducing the neutron skin. However, as there is a strong correlation between the neutron skin and the symmetry energy, we need a parametrization that produces credible results for this property.

The preliminary results obtained with the $\mathrm{DDH} \delta_{1}$ parametrization that we propose seems to produce results within the well accepted range for the main nuclear properties, including the symmetry energy. Moreover, the expected effect of the $\delta$ meson is observed, that is, a small but systematic decrease in the thickness of the neutron skin. In particular, for the ${ }^{208} \mathrm{~Pb}$ case, our DDH $\delta_{1}$ parametrization gives results close to the TW one for the energy and charge radius, but with still important differences for the skin, revealing the effect of the $\delta$ meson. As a one digit per cent ex- 
perimental error was initially expected from the PREX experiment, this could be a non-negligible effect [14].

As for the asymmetry, we may conclude that our results using a Relativistic Mean Field model show that, even for $N=Z$ nuclei, the isospin symmetry breaking has a noticeable effect, which can not be disregarded if we want to use PVE to extract precision information from the Standard Model and of the strangeness in nuclei. A closer investigation of the effects of that symmetry breaking on the strange form factor term of the asymmetry is now under way.

\section{References}

[1] M. J. Musolf, T. W. Donnelly, J. Dubach, S. J. Pollock, S. Kowalski, and E. J. Beise. Intermediate-energy semileptonic probes of the hadronic neutral current. Physics Reports, 239(1-2):1 $-178,1994$.

[2] A. Acha et al. Phys. Rev. Lett., 98(3):032301, Jan 2007.

[3] K. A. Aniol et al. Phys. Rev. Lett., 96(2):022003, Jan 2006.

[4] P. A. Souder et Al. Phys. Rev. Lett., 65(6):694-697, Aug 1990.

[5] Q-weak: A precision test of the standard model and determination of the weak charges of the quarks through parity-violating electron scattering. http://www.jlab.org/qweak/.

[6] Brian D. Serot and John D. Walecka. The Relativistic Nuclear Many-Body Problem, volume 16 of Advances in Nuclear Physics. Plenum, Nova Iorque, 1986.

[7] S. Typel and H. H. Wolter. Nuclear Physics A, 656(3-4):331 - 364, 1999.

[8] S. S. Avancini, L. Brito, J. R. Marinelli, D. P. Menezes, M. M. W. de Moraes, C. Providência, and A. M. Santos. Phys. Rev. C, 79(3):035804, Mar 2009.

[9] C. A. Graeff and J. R. Marineli. International Nuclear Physics Conference 2010 Proceedings, to be published. J. Phys.: Conf. Ser., 2011.

[10] X. Roca-Maza, M. Centelles, X. Viñas, and M. Warda. arXiv:1103.1762.

[11] G. Audi, A. H. Wapstra, and C. Thibault. Nuclear Physics A, 729(1):337 - 676, 2003. The 2003 NUBASE and Atomic Mass Evaluations.

[12] G. Fricke, C. Bernhardt, K. Heilig, L. A. Schaller, L. Schellenberg, E. B. Shera, and C. W. de Jager. At. Data Nucl. Data Tables; Vol. 60:p. 177, 1995.

[13] A. Trzcińska, J. Jastrzębski, P. Lubiński, F. J. Hartmann, R. Schmidt, T. von Egidy, and B. Kłos. Phys. Rev. Lett., 87(8):082501, Aug 2001.

[14] ${ }^{208} \mathrm{~Pb}$ Radius Experiment PREX. http://hallaweb. jlab.org/parity/prex/, 2011-09-08. 